ORIGINAL BREVE

\title{
INTENCIÓN DE LOS ESTUDIANTES DE MEDICINA DE VACUNARSE CONTRA LA GRIPE EN SU FUTURO EJERCICIO PROFESIONAL
}

\author{
Ignacio Hernández-García (1), Rafael González-Celador (2) y Mª Teresa Giménez-Júlvez (1).
}

(1) Servicio de Medicina Preventiva y Salud Pública. Hospital Universitario Infanta Leonor -Hospital Virgen de la Torre. Madrid.

(2) Departamento de Medicina Preventiva, Salud Pública y Microbiología Médica. Universidad de Salamanca. Salamanca.

Los autores declaran que no existen conflictos de interés.

\section{RESUMEN}

Fundamentos: La cobertura de vacunación antigripal en personal sanitario es baja. Este estudio se realizó con la finalidad de conocer las razones por las que los estudiantes de medicina tienen previsto vacunarse o no de gripe cuando sean trabajadores sanitarios así como los factores asociados con dicha intención.

Métodos: Estudio transversal en el que se incluyó a todos los alumnos matriculados en la Facultad de medicina de una Universidad española durante el curso académico 2011-2012. La información se obtuvo mediante un cuestionario autocumplimentado que recogía la intención de vacunación, motivos para ello y 8 preguntas sobre conocimientos de la vacuna. Se calcularon frecuencias absolutas y relativas. Para estudiar las asociaciones se utilizó la prueba chi-cuadrado y regresión logística múltiple.

Resultados: De los 1.130 estudiantes respondieron el cuestionario $654(57,9 \%)$. El $63,0 \%$ manifestaron tener intención de vacunarse. Las principales razones para prever vacunarse fueron: considerarlo conveniente $(68,2 \%)$, riesgo de transmitir gripe a pacientes $(65,5 \%)$ y riesgo de que los pacientes pudieran transmitírsela a ellos $(64,8 \%)$. Las razones más frecuentes para no prever vacunarse fueron: bajo riesgo de padecer gripe $(41,7 \%)$ y evitar inyecciones/medicación $(27,7 \%)$. Conocer específicamente que la vacuna está indicada en trabajadores sanitarios se asoció con prever vacunarse $(\mathrm{p}=0,000)$.

Conclusiones: La intención de vacunación fue elevada con respecto a la cobertura actual. El nivel de conocimientos fue mejorable. Conocer la indicación de la vacunación en trabajadores sanitarios se asoció con la intención de vacunarse.

Palabras clave: Estudiantes de medicina. Vacuna antigripal. Intención. Conocimiento. España. Personal de salud.

\section{Correspondencia}

Ignacio Hernández-García

Servicio de Medicina Preventiva y Salud Pública

Hospital Universitario Infanta Leonor -Hospital Virgen de la Torre

Gran Vía del Este, 80

28031 Madrid

ignaciohernandez79@yahoo.es

\section{ABSTRACT \\ Attitudes of Medical Students about Influenza Vaccination}

Background: Vaccination coverage against seasonal influenza virus among healthcare personnel is low. The aim of this study was to know the reasons for which the medical students have intention to be vaccinated against influenza when they become healthcare workers (HCWs), and to identify its determining factors.

Methods: A cross-sectional study was performed during the 20112012 school year. It included all enrolled medical students from a Spanish University. The information (intention to be vaccinated, reasons and 8 questions about knowledge of influenza vaccine) was obtained by a selfadministered questionnaire. Absolute and relative frequencies were calculated and the associations were then evaluated using a Chi-square test and logistic regression

Results: 654 students of the 1130 (57.9\%) completed the questionnaire. $63.0 \%$ had intention to be vaccinated. The main reasons to have intention to be vaccinated were: belief that vaccination is convenient $(68.2 \%)$, to avoid transmitting influenza to patients $(65.5 \%)$, and to avoid getting influenza from patients $(64.8 \%)$. The main reasons for not having intention to be vaccinated were: low risk of influenza $(41.7 \%)$ and to avoid shots/medication $(27.7 \%)$. Knowledge that the vaccine is recommended for HCWs was associated with the intention $(\mathrm{p}=0.000)$.

Conclusion: Intention to get vaccinated was high regarding current coverage; thus, it is needed to investigate why the coverages against influenza among Spanish physicians are usually low. The level of knowledge has left room for improvement and knowing that the vaccine is recommended for HCWs was associated with the intention.

Keyword: Students, medical. Influenza vaccines. Intention. Knowledge. Health personnel. Spain. 


\section{INTRODUCCIÓN}

La vacunación antigripal del personal sanitario constituye un elemento fundamental de los programas de prevención y control de infecciones relacionadas con la asistencia sanitaria, al ser una de las medidas más efectivas para evitar la aparición de brotes ${ }^{1}$. Pese a ello, las coberturas alcanzadas en tales trabajadores oscilan entre un 5,8\% (descrito en países del ámbito europeo tales como Grecia) ${ }^{2}$ y un $59 \%$ [porcentaje registrado en Estados Unidos (EEUU) $]^{3}$. Estas bajas frecuencias de vacunación representan un auténtico problema de salud pública, dado que el $17 \%$ de los pacientes hospitalizados con gripe adquieren la misma durante su ingreso ${ }^{4}$.

En España, la Comisión de Salud Pública del Consejo Interterritorial del Sistema Nacional de Salud recomienda la vacunación anual frente a gripe en trabajadores sanitarios (TS) pero no en estudiantes sanitarios $^{5}$, a diferencia de lo que ocurre en otros países, como EEUU, Alemania o Reino Unido, en los que diversas instituciones la recomiendan tanto en TS como en alumnos que tengan contacto con pacientes $^{6-9}$. Del mismo modo, en tales países el estudio de la utilización de la vacuna antigripal en estudiantes de ciencias de la salud ha sido objeto de varias investigaciones ${ }^{8-15}$, mientras que en España son escasos los trabajos que se han centrado en este grupo de la población ${ }^{16}$.

Dado que los alumnos sanitarios son los futuros TS, investigar sus actitudes sobre recibir o no la vacuna antigripal cuando sean trabajadores, así como sus factores determinantes, permitiría detectar elementos susceptibles de modificación mediante alguna intervención durante su periodo de formación universitaria.

El objetivo de esta investigación fue conocer la intención de los estudiantes de medicina de una universidad española sobre vacunarse o no de la gripe cuando sean TS, así como los factores que se asocian con dicha intención.

\section{MATERIAL Y MÉTODOS}

Estudio transversal desarrollado en la Universidad de Salamanca en el que se incluyó a todos los alumnos matriculados en la carrera de medicina durante el año académico 2011-2012.

Para obtener la información se utilizó un cuestionario autocumplimentado, construido a partir de los elaborados por Milunic et $\mathrm{al}^{10} \mathrm{y}$ Betsch et $\mathrm{al}^{14}$, en el que se recogieron: a) datos demográficos [sexo, edad (18-23, 24-28, 29-33, y 34 o más años), curso y nacionalidad (española y no española)]; b) intención de vacunarse cuando ejercieran su actividad profesional como TS; a su vez, se facilitó un listado de razones para justificar la intención de vacunación manifestada, pudiendo escoger más de una; c) evaluación de la importancia de internet, clases/prácticas de la carrera, médico/enfermera personal, farmacéuticos, libros de texto, artículos de revistas médicas, familiares/amigos, otros compañeros, periódicos/magazines y televisión/radio, como fuentes de información de la vacuna antigripal. Para ello se empleó una escala tipo Likert de 4 categorías $(1=$ muy poco importante; $2=$ poco importante; 3=importante; 4=muy importante); y d) evaluación de conocimientos sobre la vacuna, para lo cual se efectuaron 8 preguntas relacionadas con sus indicaciones, efectos adversos, contraindicaciones y compatibilidades (con respuestas tipo verdadero, falso o no sé).

De manera previa a la distribución del test, se hizo una prueba piloto con la participación de 3 alumnos de la Escuela Universitaria de Enfermería y Fisioterapia de la Universidad de Salamanca, así 
como con la de un facultativo y un médico interno residente del Servicio de Medicina Preventiva del Complejo Asistencial de Salamanca, a quienes se pidió que evaluaran su legibilidad y comprensibilidad con el fin de ratificar que el cuestionario era legible y de fácil comprensión a la hora de ser respondido.

Durante la segunda semana de febrero de 2012, profesores de la Universidad repartieron los tests entre el alumnado presente en el momento de realización del estudio. En cada curso los cuestionarios se pasaron una sola vez, entregándose y recogiéndose en los minutos finales de las clases en las que mayor asistencia solía haber. Asimismo, los mismos profesores explicaron el objetivo del estudio y la forma de cumplimentar el test, informando también del carácter confidencial, voluntario y anónimo de la investigación.

Se calcularon frecuencias absolutas y relativas (porcentajes) para las distintas variables y se evaluó la existencia de diferencias según el curso (categorizado en: a) $1^{\circ}-2^{\circ}$; b) $3^{\circ}-4^{\circ}$; y c) $5^{\circ}-6^{\circ}$ ). Además, se realizó un análisis univariante en el que se consideró variable resultado el tener o no intención de vacunarse de gripe cuando ejercieran como TS y como variables explicativas la edad, sexo, curso, nacionalidad, conocimientos e importancia de las distintas fuentes de información de la vacuna. Para ello se utilizó la prueba chi $^{2}$ y para cuantificar la asociación se calcularon las odds ratio (OR) y sus intervalos de confianza del 95\% (IC95\%). Posteriormente se realizó un análisis de regresión logística múltiple con las variables en las que se observó asociación significativa en el análisis univariante.

El nivel de significación estadística considerado en todos los contrastes de hipótesis fue $\mathrm{p}<0,05$ y el programa de análisis empleado el SPSS v20.0.

\section{RESULTADOS}

El número de estudiantes que respondieron el cuestionario fue $654(57,9 \%)$ de los 1.130 alumnos matriculados en alguno de los seis cursos de la carrera de medicina y $82,4 \%$ de los 794 alumnos presentes en clase en el momento en el que se distribuyó el cuestionario. $455(69,6 \%)$ de los 654 estudiantes eran mujeres, con una edad de entre 18 y 23 años en el $89,0 \%$ de los casos.

De los 654 alumnos $412(63,0 \%)$ manifestaron tener intención de recibir la vacuna antigripal cuando fueran TS $\left(1^{\circ}\right.$ y $2^{\circ}$ curso: $184(62,6 \%)$ de $294 ; 3^{\circ}$ y $4^{\circ}$ curso $(61,1 \%)$ de $198 ; 5^{\circ}$ y $6^{\circ}$ curso: 107 $(66,0 \%)$ de $162 ; p=0,615)$. Por su parte, los restantes $242(37,0 \%)$ estudiantes respondieron que no tenían intención de vacunarse cuando ejercieran.

Entre las razones alegadas para tener intención de vacunarse destacaron: considerarlo conveniente $(68,2 \%)$, el riesgo de poder transmitir la gripe a los pacientes $(65,5 \%)$ y el riesgo de que los pacientes pudieran transmitirsela a ellos $(64,8 \%)$. Asimismo, según el curso, se observaron diferencias estadísticamente significativas en varios de los motivos, en particular considerar la efectividad de la vacuna como motivo para tener intención de vacunarse fue el que más difirió, existiendo una diferencia de hasta 18,1 puntos porcentuales entre los alumnos de $5^{\circ}-6^{\circ}$ con respecto a los de $1^{\circ}-2^{\circ}$ curso (tabla 1 ).

Las principales razones para justificar no prever vacunarse fueron: considerarse en bajo riesgo de padecer la gripe $(41,7 \%)$, evitar inyecciones/medicación innecesaria $(27,7 \%)$ y dudas sobre la efectividad de la vacuna $(17,4 \%)$. En concreto, la inconveniencia/estar demasiado ocupado como motivo para no tener intención de vacunarse fue la única razón en la que se detectaron diferencias esta- 
Tabla 1

Razones alegadas para tener intención o no de vacunarse frente a la gripe cuando sean trabajadores sanitarios según el curso

\begin{tabular}{|c|c|c|c|c|}
\hline & $\begin{array}{c}1^{\circ}-2^{\circ} \text { curso } \\
n(\%)\end{array}$ & $\begin{array}{c}3^{\circ}-4^{\circ} \text { curso } \\
n(\%)\end{array}$ & $\begin{array}{c}5^{\circ}-6^{\circ} \text { curso } \\
\text { n }(\%)\end{array}$ & $\mathrm{p}$ \\
\hline \multicolumn{5}{|l|}{ Razones alegadas para tener intención de vacunarse: } \\
\hline - Considero que es conveniente vacunarme & $126(68,5)$ & $85(70,2)$ & $70(65,4)$ & 0,733 \\
\hline - Riesgo de que pueda transmitir la gripe a los pacientes & $127(69,0)$ & $67(55,4)$ & $76(71,0)$ & 0,019 \\
\hline - Riesgo de que pacientes me puedan transmitir la gripe & $132(71,7)$ & $76(62,8)$ & $59(55,1)$ & 0,015 \\
\hline $\begin{array}{l}\text { - Cumplir con la política de vacunación del hospital, } \\
\text { centro de salud o clínica privada donde ejerza } \\
\text { - Riesgo de que pueda transmitir la gripe a mi familia o a }\end{array}$ & $66(35,9)$ & $51(42,1)$ & $44(41,1)$ & 0,481 \\
\hline las personas con las que conviva & $84(45,7)$ & $36(29,8)$ & $39(36,4)$ & 0,000 \\
\hline - La vacuna es efectiva & $39(21,2)$ & $46(38,0)$ & $42(39,3)$ & 0,001 \\
\hline - Me han recomendado vacunarme & $19(10,3)$ & $28(23,1)$ & $23(21,5)$ & 0,005 \\
\hline - La vacuna es gratuita & $11(6,0)$ & $13(10,7)$ & $18(16,8)$ & 0,013 \\
\hline - La vacuna me protegió de la gripe en el pasado & $5(2,7)$ & $10(8,3)$ & $7(6,5)$ & 0,088 \\
\hline - Padecer una enfermedad crónica & $4(2,2)$ & $8(6,6)$ & $9(8,4)$ & 0,044 \\
\hline -Haber tenido la gripe anteriormente & $4(2,2)$ & $9(7,4)$ & $4(3,7)$ & 0,076 \\
\hline Total & 184 & 121 & 107 & \\
\hline \multicolumn{5}{|l|}{ Razones alegadas para no tener intención de vacunarse: } \\
\hline - Bajo riesgo de padecer la gripe & $49(44,5)$ & $29(37,7)$ & $23(41,8)$ & 0,643 \\
\hline - Evitar pinchazos o medicación innecesaria & $29(26,4)$ & $28(36,4)$ & $10(18,2)$ & 0,065 \\
\hline - La vacuna no es efectiva & $17(15,5)$ & $19(24,7)$ & $6(10,9)$ & 0,093 \\
\hline - Se me olvidará & $18(16,4)$ & $8(10,4)$ & $8(14,5)$ & 0,508 \\
\hline - Miedo a los efectos adversos de la vacuna & $15(13,6)$ & $10(13,0)$ & $6(10,9)$ & 0,884 \\
\hline - Inconveniencia / Estaré demasiado ocupado & $5(4,5)$ & $6(7,8)$ & $11(20,0)$ & 0,005 \\
\hline - En el pasado tuve la gripe pese a haberme vacunado & $8(7,3)$ & $10(13,0)$ & $2(3,6)$ & 0,138 \\
\hline - Coste de la vacunación & $6(5,5)$ & $3(3,9)$ & $2(3,6)$ & 0,823 \\
\hline - Contraindicación médica & $2(1,8)$ & $3(3,9)$ & $0(0)$ & 0,291 \\
\hline - No saber dónde conseguir la vacuna & $1(0,9)$ & $1(1,3)$ & $1(1,8)$ & 0,882 \\
\hline Total & 110 & 77 & 55 & \\
\hline
\end{tabular}

Resultados de las respuestas expresados como frecuencias absolutas (n) y relativas (\%).

dísticamente significativas entre distintos cursos (tabla 1).

Por lo que respecta a los conocimientos evaluados 104 (15,9\%) de los 654 estudiantes contestaron adecuadamente a $5 \mathrm{o}$ más de las 8 preguntas efectuadas, existiendo diferencias estadísticamente significativas según el curso $\left(1^{\circ}-2^{\circ}\right.$ curso: 17 de $294(5,8 \%) ; 3^{\circ}-4^{\circ}$ curso: 20 de 198 $(10,1 \%) ; 5^{\circ}-6^{\circ}$ curso: 67 de $162(41,4 \%)$; $\mathrm{p}=0,000)$. El mayor porcentaje de res- puestas correctas se obtuvo para los ítems "conocer que la vacuna está indicada en TS" $(61,0 \%)$ y "no puede causar la gripe" $(43,7 \%)$. Por su parte, considerar que la lactancia y la inmunosupresión no representan una contraindicación para recibir la vacuna fue respondido de manera inadecuada por el $83,2 \%$ y $80,4 \%$ del alumnado, respectivamente. Según el curso, hubo diferencias estadísticamente significativas en todos los conocimientos estudiados, principalmente en los ítems 
Tabla 2

Conocimientos de los estudiantes de medicina según el curso

\begin{tabular}{|c|c|c|c|c|}
\hline & \begin{tabular}{c|}
$1^{\circ}-2^{\circ}$ curso \\
$(n=294)$
\end{tabular} & $\begin{array}{c}3^{\circ}-4^{\circ} \text { curso } \\
(n=198)\end{array}$ & $\begin{array}{c}5^{\circ}-6^{\circ} \text { curso } \\
(\mathrm{n}=162)\end{array}$ & $\mathrm{p}$ \\
\hline \multicolumn{5}{|l|}{ La vacuna de la gripe está indicada en todos los trabajadores de centros sanitarios } \\
\hline Verdadero* & $160(54,4)$ & $116(58,6)$ & $123(75,9)$ & 0,000 \\
\hline Falso & $134(45,6)$ & $82(41,4)$ & $39(24,1)$ & \\
\hline \multicolumn{5}{|l|}{ Una persona puede contraer la gripe a través de la vacuna } \\
\hline Verdadero & $193(65,6)$ & $110(55,6)$ & $65(40,1)$ & 0,000 \\
\hline Falso* & $101(34,4)$ & $88(44,4)$ & $97(59,9)$ & \\
\hline \multicolumn{5}{|c|}{\begin{tabular}{l|l|l|l} 
¿Cuál/es de las siguientes situaciones contraindica recibir la vacuna antigripal? & & \\
Lactancia & &
\end{tabular}} \\
\hline Verdadero & $267(90,8)$ & $173(87,4)$ & $104(64,2)$ & 0,000 \\
\hline Falso* & $27(9,2)$ & $25(12,6)$ & $58(35,8)$ & \\
\hline \multicolumn{5}{|l|}{ Alergia al huevo } \\
\hline Verdadero* & $84(28,6)$ & $34(17,2)$ & $54(33,3)$ & 0,001 \\
\hline Falso & $210(71,4)$ & $164(82,8)$ & $108(66,7)$ & \\
\hline \multicolumn{5}{|l|}{ Infección leve del tracto respiratorio superior } \\
\hline Verdadero & $243(82,7)$ & $154(77,8)$ & $79(48,8)$ & 0,000 \\
\hline Falso* & $51(17,3)$ & $44(22,2)$ & $83(51,2)$ & \\
\hline \multicolumn{5}{|l|}{ Embarazo } \\
\hline Verdadero & $263(89,5)$ & $173(87,4)$ & $84(51,9)$ & 0,000 \\
\hline Falso* & $31(10,5)$ & $25(12,6)$ & $78(48,1)$ & \\
\hline \multicolumn{5}{|l|}{ Enfermedad febril aguda grave } \\
\hline Verdadero* & $89(30,3)$ & $87(43,9)$ & $99(61,1)$ & 0,000 \\
\hline Falso & $205(69,7)$ & $111(56,1)$ & $63(38,9)$ & \\
\hline \multicolumn{5}{|l|}{ Inmunosupresión } \\
\hline Verdadero & $254(86,4)$ & $165(83,3)$ & $107(66,1)$ & 0,000 \\
\hline Falso* & $40(13,6)$ & $33(16,7)$ & $55(33,9)$ & \\
\hline
\end{tabular}

Resultados de las respuestas expresados como frecuencias absolutas (n) y relativas (\%). * Respuesta correcta.

"conocer que ni el embarazo ni las infecciones leves del tracto respiratorio superior suponen una contraindicación", en los que los porcentajes de respuestas adecuadas fueron, respectivamente, 37,6 y 33,9 puntos más altos en alumnos de $5^{\circ}-6^{\circ}$ que en los de $1-2^{\circ}$ curso (tabla 2 ).

En cuanto a la importancia de las fuentes información sobre la vacuna fueron valoradas como muy importantes las clases/prácticas $(17,9 \%)$, médico/a y enfermera/o personal $(15,6 \%)$, los artículos de revistas médicas $(12,8 \%)$, libros de texto $(11,6 \%)$, familiares/amigos $(8,8 \%)$, internet $(8,4 \%)$, TV/radio $(7,2 \%)$, farmacéuti$\cos (4,4 \%)$, periódicos/magazines $(2,9 \%)$ y otros compañeros $(1,0 \%)$.
Los resultados del estudio de asociación univariante entre las características de los alumnos, sus conocimientos, y la intención de vacunarse cuando fueran TS se recogen en la tabla 3 . Conocer que la vacuna está indicada en TS se asoció significativamente con la intención de vacunarse (OR:2,2;IC95\%:1,6-3,0). Otros factores que también se asociaron con dicha intención fueron: valorar como fuentes de información muy importantes tanto a los artículos científicos como a los farmacéuticos (OR: 1,8 (IC95\%: 1,0$3,2)$; $\mathrm{p}=0,043$ y OR: 6,8 (IC95\%: 1,6$29,5) ; p=0,006$, respectivamente) y responder adecuadamente al menos a 5 de las 8 preguntas de conocimientos (OR: 1,7 (IC95\%: 1,1-2,8); $\mathrm{p}=0,027)$. Los 


\section{Tabla 3}

Estudio de asociación entre características de los alumnos, conocimientos sobre vacuna antigripal e intención de vacunarse cuando sean trabajadores sanitarios

\begin{tabular}{|c|c|c|c|c|}
\hline & \multicolumn{2}{|c|}{ Intención de vacunarse } & \multirow{2}{*}{$\begin{array}{c}\text { OR } \\
(\mathrm{IC} 95 \%)^{*}\end{array}$} & \multirow{2}{*}{$\mathrm{p}$} \\
\hline & Sí (n=412) & No $(n=242)$ & & \\
\hline \multicolumn{5}{|c|}{ Edad (años) - \% (n) } \\
\hline $18-23$ & $89,3 \%(368)$ & $88,4 \%(214)$ & $1,1(0,7-1,8)$ & 0,824 \\
\hline$>24$ & $10,7 \%(44)$ & $11,6 \%(28)$ & 1 & \\
\hline \multicolumn{5}{|l|}{ Sexo - \% (n) } \\
\hline Hombre & $30,6 \%(126)$ & $30,2 \%(73)$ & $1,0(0,7-1,4)$ & 0,981 \\
\hline Mujer & $69,4 \%(286)$ & $69,8 \%(169)$ & 1 & \\
\hline \multicolumn{5}{|c|}{ Nacionalidad - \% (n) } \\
\hline Española & $85,2 \%(351)$ & $86,8 \%(210)$ & $0,9(0,6-1,4)$ & 0,657 \\
\hline Otras & $14,8 \%(61)$ & $13,2 \%(32)$ & 1 & \\
\hline \multicolumn{5}{|l|}{ Curso - \% (n) } \\
\hline Curso $5^{\circ}-6^{\circ}$ & $26,0 \%(107)$ & $22,7 \%(55)$ & $1,2(0,8-1,8)$ & 0,462 \\
\hline Curso $3^{\circ}-4^{\circ}$ & $29,4 \%(121)$ & $31,8 \%(77)$ & $0,9(0,6-1,4)$ & 0,741 \\
\hline Curso $1^{\circ}-2^{\circ}$ & $44,7 \%(184)$ & $45,5 \%(110)$ & 1 & \\
\hline \multicolumn{5}{|c|}{ Conocer indicación en TS - \% (n) } \\
\hline Sí & $68,0 \%(280)$ & $49,2 \%(119)$ & $2,2(1,6-3,0)$ & 0,000 \\
\hline No & $32,0 \%(132)$ & $50,8 \%(123)$ & 1 & \\
\hline \multicolumn{5}{|c|}{ Conocer que no causa gripe - \% (n) } \\
\hline Sí & $45,4 \%(187)$ & $40,9 \%(99)$ & $1,2(0,9-1,7)$ & 0,301 \\
\hline No & $54,6 \%(225)$ & $59,1 \%(143)$ & 1 & \\
\hline \multicolumn{5}{|c|}{$\begin{array}{l}\text { Conocer contraindicación en: } \\
\text { - Alérgicos al huevo - \% (n) }\end{array}$} \\
\hline Sí & $26,0 \%(107)$ & $26,9 \%(65)$ & $0,9(0,7-1,4)$ & 0,875 \\
\hline No & $74,0 \%(305)$ & $73,1 \%(177)$ & 1 & \\
\hline \multicolumn{5}{|c|}{ - Enfermedad febril grave - \% (n) } \\
\hline Sí & $40,3 \%(166)$ & $45,0 \%(109)$ & $0,8(0,6-1,1)$ & 0,269 \\
\hline No & $59,7 \%(246)$ & $55,0 \%(133)$ & 1 & \\
\hline \multicolumn{5}{|c|}{ Conocer compatibilidad con: } \\
\hline \multicolumn{5}{|c|}{ - Embarazo - \% (n) } \\
\hline Sí & $21,8 \%(90)$ & $18,2 \%(44)$ & $1,3(0,8-1,9)$ & 0,308 \\
\hline No & $78,2 \%(322)$ & $81,8 \%(198)$ & 1 & \\
\hline \multicolumn{5}{|c|}{ - Lactancia - \% (n) } \\
\hline Sí & $18,9 \%(78)$ & $13,2 \%(32)$ & $1,5(0,9-2,4)$ & 0,076 \\
\hline No & $81,1 \%(334)$ & $86,8 \%(210)$ & 1 & \\
\hline \multicolumn{5}{|c|}{ - Inmunosupresión - \% (n) } \\
\hline Sí & $19,9 \%(82)$ & $19,0 \%(46)$ & $1,1(0,7-1,6)$ & 0,860 \\
\hline No & $80,1 \%(330)$ & $81,0 \%(196)$ & 1 & \\
\hline \multicolumn{5}{|c|}{ - Infección respiratoria leve -\% (n) } \\
\hline Sí & $26,9 \%(111)$ & $27,7 \%(67)$ & $0,9(0,7-1,4)$ & 0,908 \\
\hline No & $73,1 \%(301)$ & $72,3 \%(175)$ & 1 & \\
\hline
\end{tabular}

Resultados expresados como frecuencias relativas (\%) y absolutas (n). * OR (IC 95\%): Odds Ratio (Intervalo de Confianza del 95\%).

resultados del análisis de regresión logística evidenciaron cómo las variables que finalmente se asociaron significativamente con la intención de vacunación fueron conocer que está indicada en TS
[OR: 2,3 (IC95\%: 1,6-3,3), p=0,000)] y estimar a los farmacéuticos como muy importantes fuentes de información de la vacuna antigripal [OR: 6,2 (IC95\%: 1,426,8), $\mathrm{p}=0,016)]$. 


\section{DISCUSIÓN}

Más de la mitad de los estudiantes manifestaron tener intención de vacunarse durante su futuro ejercicio profesional. La principal razón para ello fue considerarlo conveniente mientras que el motivo más común para no prever vacunarse fue percibir un riesgo bajo de padecer gripe. Conocer que la vacuna está indicada en TS se asoció con la intención de vacunarse.

Este trabajo es, hasta nuestro conocimiento, el único que ha investigado las razones por las que estudiantes de todos los cursos de la carrera de medicina de una universidad española tienen previsto vacunarse o no de gripe cuando sean TS, pues, hasta la fecha, dicha investigación se ha centrado exclusivamente en estudiantes de último curso de diferentes carreras sanitarias ${ }^{16}$.

La particularidad de que hayamos estudiado la intención de vacunarse cuando fueran TS, en lugar de determinar la intención que presentaban con respecto a recibir la vacuna durante la siguiente temporada gripal, o conocer cuál era el porcentaje de estudiantes que se habían vacunado en la última temporada, tal como han hecho diversos autores en otras partes del mundo ${ }^{9,11,14,17}$, se debe a que en España, la Comisión de Salud Pública del Consejo Interterritorial del Sistema Nacional de Salud recomienda la vacunación anual frente a gripe en TS pero no en estudiantes sanitarios ${ }^{5}$.

De acuerdo a este matiz, la intención de vacunación, que detectamos en nuestros estudiantes, fue más alta que la documentada en determinados países, tales como Alemania o Reino Unido, donde Betsch et $\mathrm{al}^{14}$ en alumnos de medicina de la Universidad de Frankfurt, y Hunt et $\mathrm{al}^{9}$ en alumnos de enfermería de la Universidad de Nottingham, describieron cómo hasta el 80,2\% de tales estudiantes no tenían intención de vacunarse frente a la gripe durante la siguiente temporada ${ }^{9}$. Por su parte, la inten- ción manifestada por nuestro alumnado se situó en una posición intermedia con respecto a la frecuencia de vacunación registrada en estudiantes de medicina de EEUU $^{10-12}$, en los que dicha cobertura oscila entre un $48 \%{ }^{10}$ y un $92 \%{ }^{12}$. Finalmente, la intención detectada se halla considerablemente por encima de la frecuencia de vacunación documentada en España ${ }^{18-20}$, donde las coberturas obtenidas en los últimos años se han cuantificado entre un $11,2 \%{ }^{18}$ y un $35,0 \%$ en personal sanitario en general $^{20} \mathrm{y}$ entre un $19,2 \%{ }^{18}$ y un $38,1 \%{ }^{19}$ en personal facultativo en particular.

Esta sorprendente discrepancia entre la intención de vacunación de nuestros estudiantes y las coberturas alcanzadas en TS de nuestro país podría justificarse por la existencia de un sesgo por conveniencia social (social desirability bias), dado que los alumnos podrían haber decido seleccionar la respuesta considerada como la socialmente más aceptable ${ }^{21}$. No obstante, no haber empleado la entrevista personal para recoger la información, al haberse utilizado, en su lugar, un cuestionario anónimo autocumplimentado, en el que, además, en ningún caso se recogía información que pudiera quebrantar el mencionado anonimato de los respondedores (al no registrarse el nombre/apellidos, la fecha de nacimiento, o la nacionalidad de los alumnos no españoles) constituye un modo de haber controlado la presencia de dicho potencial sesgo ${ }^{21}$.

Asimismo, la mencionada discrepancia resulta difícil de explicar, sobre todo si tenemos en cuenta los resultados de los escasos trabajos que han investigado la relación intención-comportamiento para la vacunación antigripal en $\mathrm{TS}^{22}$. Así, Godin et $\mathrm{al}^{22}$, en un estudio realizado para establecer qué factores determinan que el personal sanitario se vacune o no de la gripe, observaron cómo ajustando por la variable comportamiento previo la intención constituía el principal factor seguido del sentimiento de remordimiento, la moralidad y la situación laboral 
(jornada completa o parcial). Dado que, tal y como señala dicho autor, la mayoría de las personas actúan de acuerdo a su intención, y dado que en nuestro medio no existe información específica sobre la cobertura de vacunación antigripal en personal sanitario de nueva incorporación al mundo laboral, se hace necesario realizar una investigación para conocer dicha cobertura. De este modo, en caso de que fuera igual de baja que las registradas hasta ahora en TS en general, se debería estudiar qué sucede en el entorno laboral de nuestro país para que personas con una intención de vacunación a priori tan alta no se vacunen cuando ejercen su actividad profesional.

Por lo que respecta a las razones manifestadas, para tener intención de vacunarse, destacaron cómo las tres más frecuentes por al menos el $64 \%$ del total de alumnos la conveniencia, el riesgo de transmitir gripe a los pacientes y el riesgo de que los pacientes pudieran transmitírsela. Estas razones presentan la particularidad de encontrarse entre las principales por las que los estudiantes de medicina se vacunan de la gripe en otros países ${ }^{8,10,11}$. Asimismo, el que hayamos detectado cómo más de la mitad aludió al riesgo de poder transmitir la gripe a los pacientes, probablemente indica falta de conocimientos en el resto de estudiantes en cuanto al papel del profesional sanitario en la transmisión nosocomial del virus de la gripe, hallazgo relevante pues el motivo principal, para recomendar la vacunación en TS es proteger la salud de los pacientes a los que atienden ${ }^{6}$.

Otros motivos frecuentes para prever vacunarse fueron el riesgo de sufrir gripe o transmitirla a su familia. Por ello, una posible estrategia para mejorar dicha intención sería incluir información específica sobre gripe y su vacuna en TS en el temario del programa docente de la carrera de medicina. En él se haría constar la susceptibilidad del trabajador frente a este virus y la posibilidad de trasmitirlo tanto a los pacientes que atienda como a las personas que convivan con él. Además, todo tipo de personal sanitario (médico, enfermería y farmacéutico) con el que se hacen las prácticas debería informar a los estudiantes así como indicarles que se vacunen cuando sean TS, dado que: a) conocer tal indicación se asoció con la intención de recibir la vacuna; b) las clases/prácticas fueron frecuentemente consideradas como las fuentes de información más importantes para recibir información específica sobre la vacuna; y c) confiar en la información que pueden aportar determinados TS (farmacéuticos) se asoció con una mayor intención de vacunarse.

La efectividad de la vacuna destacó por ser un motivo citado tanto por los que tenían intención de vacunarse como por los que no, principalmente en alumnos de $3^{\circ}-4^{\circ}$ curso. Cuestionar la efectividad manifiesta un déficit de conocimientos sobre este aspecto, ya que previene el $70-90 \%$ de las gripes en adultos sanos menores de 65 años si la cepa del virus incluido coincide con la circulante $^{5}$. Dado que su efectividad disminuye en ancianos y personas inmunodeprimidas $^{5}$, vacunar al TS representa un modo de proteger indirectamente a estos grupos de población, aspecto destacable ya que sólo el 52,6\% de los pacientes mayores de 65 años con enfermedades crónicas que ingresan en hospitales están vacunados ${ }^{23}$.

En cuanto a las razones manifestadas para no prever vacunarse, se observó una gran heterogeneidad en las respuestas obtenidas, sin que ninguna fuese seleccionada por más del $42 \%$ del alumnado. Este hallazgo es en sí mismo importante, ya que revela la complejidad de la situación e indica cómo se precisa proporcionar formación diversa para rebatir cada una de ellas. En particular, percibir un riesgo bajo de padecer gripe (principal razón para no tener intención de vacunarse) demostró, nuevamente, cómo algunos alumnos no reconocen el papel del TS en la transmisión nosocomial del virus y por ello se hace preciso 
que durante su formación universitaria se incida específicamente sobre la responsabilidad ética que tiene el personal sanitario en cuanto a proteger a sus pacientes de sufrir una gripe nosocomial ${ }^{24}$.

Por su parte, el evitar inyecciones/medicación innecesaria y el miedo a los efectos adversos (EA) (frecuentes motivos por las que tanto estudiantes ${ }^{9-11}$ como TS no se vacunan $^{11,17,25}$ ) también destacaron como razones para no prever vacunarse. Sin embargo, dada la seguridad de la vacuna $^{5,26,27}$, ningún alumno debería justificar su intención de no vacunarse por temor a los EA. Por este motivo, en su formación se debería informar sobre posibles EA y sobre su benignidad, señalando que no puede causar gripe, habida cuenta que dicho error de conocimiento se detectó con frecuencia.

Del mismo modo, se hace preciso proporcionar y/o reforzar la formación que específicamente se imparte sobre la vacuna antigripal en la facultad para subsanar los restantes errores de conocimientos que se detectan, sobre todo porque, tal y como hemos observado para determinados conocimientos con respecto a la intención, varios autores han demostrado cómo un mejor nivel de conocimientos se asocia con una mayor frecuencia de vacunación en estudiantes ${ }^{10} \mathrm{y}$ en personal sanitario ${ }^{12}$. A este respecto, la importancia de educar para conseguir buenas coberturas vacunales se ha minusvalorado argumentando que proporcionar formación no asegura la comprensión $^{28}$. Sin embargo, durante los años de carrera puede asegurarse la comprensión al evaluarla mediante exámenes. Además, tales años representan una excelente oportunidad de llegar a todos, aspecto que los programas formativos de TS no consiguen, como demuestra la escasa asistencia que suele haber a los cursos de formación continuada.

En cualquier caso, pese a que los conocimientos mejoraron durante la carrera, hallazgo que ha sido descrito también por otros autores, como Milunic et $\mathrm{al}^{10}$, están lejos de poder considerarse como aceptables. En particular, el mencionado hecho de haber detectado conocimientos mejorables, similar a lo obtenido por otros autores en investigaciones llevadas a cabo en países de nuestro entorno, como Alemania, donde hasta el $36,5 \%$ de los estudiantes de medicina no conocían la recomendación oficial de que los TS reciban la vacuna antigripal ${ }^{14}$, es relevante, sobre todo cuando incluso los alumnos de los últimos cursos presentaron conocimientos muy mejorables. Este hallazgo es importante, porque la falta de conocimientos con los que los estudiantes finalizan sus estudios puede contribuir al mantenimiento de las bajas coberturas vacunales que se registran en nuestro país, ya no solo en TS sino en población general, dado que difícilmente un facultativo va a recomendar a sus pacientes que se vacunen de la gripe si él mismo no conoce las indicaciones y/o contraindicaciones correspondientes. Por su parte, en España, la cobertura vacunal en mujeres embarazadas se ha cuantificado en un $5,7 \%{ }^{29}$.

Entre las limitaciones de nuestro trabajo se encuentra haber sido realizado en una sola universidad, situación que motiva que se precisen desarrollar estudios similares para establecer si las necesidades formativas detectadas son iguales en el resto del país, lo cual es previsible dada la semejanza de los programas formativos. Asimismo, debido a que el trabajo se realizó exclusivamente en alumnos de medicina, no podemos generalizar los resultados al total de estudiantes sanitarios, siendo necesario llevar a cabo investigaciones específicas en los restantes alumnos de ciencias de la salud. En particular, consideramos que tales estudios se deberían hacer en todos los cursos dado que permitiría detectar necesidades formativas específicas potencialmente subsanables mediante las correspondientes intervenciones formativas a desarrollar en los distintos cursos y durante los años de formación universitaria. 
Finalmente, pese a que las 654 personas que respondieron al cuestionario constituyeron la mayor serie de alumnos de medicina estudiados en todo el mundo, respecto a la intención o frecuencia de vacunación y conocimientos que poseen sobre dicha vacuna antigripal, y a pesar de que la tasa de respuesta fue mejor que la obtenida por otros autores, tales como Milunic et al $(41 \%)^{10}$ o Wicker et al $(23,8 \%)^{8}$, no podemos descartar la presencia de un posible sesgo por no respuesta, habitual en este tipo de investigaciones ${ }^{30}$.

Con nuestro trabajo se muestra la necesidad de plantear una nueva forma de afrontar el problema de alcanzar adecuadas coberturas vacunales en TS, anticipándonos a su aparición al intervenir en estudiantes, impartiendo formación específica sobre gripe y su vacuna y evaluandola. Con ello planteamos una nueva forma con la que contribuir a mejorar dichas coberturas en nuestro medio, ya que es con la anticipación a la aparición de los problemas el modo con el que otras políticas de salud pública han logrado ser efectivas en España ${ }^{31}$. Además, dado que los estudiantes de medicina entran en contacto con pacientes durante su periodo de formación universitaria, pudiendo actuar como vectores en la transmisión del virus de la gripe ${ }^{10} \mathrm{y}$ dado que la variable comportamiento previo (es decir, haber recibido la vacuna en el pasado) constituye un importante determinante de la vacunación antigripal ${ }^{22}$, también estimamos necesario que se implementen intervenciones para estimular a los alumnos a vacunarse por primera vez como primer paso para generar el hábito de vacunarse posteriormente todos los años. Por ello consideramos imprescindible que la Comisión de Salud Pública del Consejo Interterritorial del Sistema Nacional de Salud de nuestro país se planteé incluir a este colectivo de población en los grupos en los que recomienda que se vacunen anualmente de la gripe. De este modo, se aprovecharía que su intención se lleve a la práctica, generándoles así el hábito que podrían mantener cuando fueran profesionales, con lo cual se mejorarían las futuras coberturas de vacunación en personal sanitario.

\section{AGRADECIMIENTOS.}

Al profesorado del Departamento de Medicina Preventiva, Salud Pública y Microbiología Médica de la Universidad de Salamanca, por su colaboración en la distribución del cuestionario; en especial, al Dr. Rafael González por su amistad y apoyo profesional a lo largo de estos últimos 10 años.

\section{BIBLIOGRAFÍA}

1. Loulergue P, Moulin F, Vidal-Trecan G, Absi Z, Demontpion C, Menager C, et al. Knowledge, attitudes and vaccination coverage of healthcare workers regarding occupational vaccinations. Vaccine. 2009;27:4240-3.

2. Maltezou HC, Maragos A, Katerelos P, Paisi A, Karageorgou K, Papadimitriou $\mathrm{T}$, et al. Influenza vaccination acceptance among health-care workers: a nationwide survey. Vaccine. 2008;26:1408-10.

3. Gazmararian JA, Coleman M, Prill M, Hinman $\mathrm{AR}$, Ribner BS, Washington $\mathrm{ML}$, et al. Influenza vaccination of health care workers: policies and practices of hospitals in a community setting. Am J Infect Control. 2007;35:441-7.

4. Adal KA, Flowers RH, Anglim AM, Hayden FG, Titus MG, Coyner BJ, et al. Prevention of nosocomial influenza. Infect Control Hosp Epidemiol. 1996;17:641-8.

5. Ministerio de Sanidad, Servicios Sociales e Igualdad. Prevención de la Gripe. Vacunación antigripal. [Citado el 30 de noviembre de 2013]. Disponible en: http://msc.es/ciudadanos/enfLesiones/enfTransmisibles/gripe/gripe.htm\#prevencion

6. Fiore AE, Shay DK, Broder K, Iskander JK, Uyeki TM, Mootrey G, et al; Centers for Disease Control and Prevention. Prevention and control of seasonal influenza with vaccines: recommendations of the Advisory Committee on Immunization Practices (ACIP), 2009. MMWR Recomm Rep. 2009;58(RR8):1-52. 
7. Centers for Disease Control and Prevention (CDC). Prevention and control of seasonal influenza with vaccines. Recommendations of the Advisory Committee on Immunization Practices--United States, 2013-2014. MMWR Recomm Rep. 2013;62(RR-07):1-43.

8. Wicker S, Rabenau HF, von Gierke L, François G, Hambach R, De Schryver A. Hepatitis B and influenza vaccines: important occupational vaccines differently perceived among medical students. Vaccine. 2013;31:5111-7.

9. Hunt C, Arthur A. Student nurses' reasons behind the decision to receive or decline influenza vaccine: a cross-sectional survey. Vaccine. 2012;30:5824-9.

10. Milunic SL, Quilty JF, Super DM, Noritz GH. Patterns of influenza vaccination among medical students. Infect Control Hosp Epidemiol. 2010;31:85-8.

11. Christini AB, Shutt KA, Byers KE. Influenza vaccination rates and motivators among healthcare worker groups. Infect Control Hosp Epidemiol. 2007;28:171-7.

12. Martinello RA, Jones L, Topal JE. Correlation between healthcare workers' knowledge of influenza vaccine and vaccine receipt. Infect Control Hosp Epidemiol. 2003;24:845-7.

13. Ali S, Khakoo R, Fisher M, Hobbs GR. An assessment of influenza vaccination among health profession students. Scand J Infect Dis. 2007;39:822-5.

14. Betsch C, Wicker S. E-health use, vaccination knowledge and perception of own risk: drivers of vaccination uptake in medical students. Vaccine. 2012;30:1143-8.

15. Afonso N, Kavanagh M, Swanberg S. Improvement in attitudes toward influenza vaccination in medical students following an integrated curricular intervention. Vaccine. 2014;32:502-6.

16. Hernández-García I, Cardoso-Muñoz A, MorenoPascual C, Sáenz-González MC. Razones de los estudiantes sanitarios para tener intención de vacunarse o no de gripe cuando sean trabajadores sanitarios. Enferm Clin. 2011;21:300-1.

17. Askarian M, Khazaeipour Z, McLaws ML. Influenza vaccination uptake among students and clinical staff of a university in Iran. Int J Infect Dis. 2009;13:476-82.

18. del Villar-Belzunce A, Hernández-Navarrete MJ, Lapresta-Moros C, Solano-Bernad VM, Arribas-Llorente JL. Vacunación antigripal en personal sanitario. Enferm Infecc Microbiol Clin. 2007;25:247-9.
19. Sánchez-Payá J, Hernández-García I, Barrenengoa Sañudo J, Rolando Martínez H, Camargo Ángeles $\mathrm{R}$, Cartagena Llopis L, et al. Determinantes de la vacunación antigripal en personal sanitario, temporada 2009-2010. Gac Sanit. 2011;25:29-34.

20. García de Codes A, Arrazola MP, de Juanes JR, Hernández MT, Jaén F, Sanz I. Campaña de vacunación antigripal (pandémica y estacional) en trabajadores de un hospital general (2009-2010). Vacunas. 2010;11:49-53.

21. Ipsos MORI Scotland. Four ways to get the truth out of respondents. [Citado el 23 de diciembre de 2013]. Disponible en: http://www.ipsosmori.com/contactus/offices/scotland/approachnewsletter/Approach-Autumn-2012.aspx

22. Godin G, Vézina-Im LA, Naccache H. Determinants of influenza vaccination among healthcare workers. Infect Control Hosp Epidemiol. 2010;31:68993.

23. Sánchez Muñoz-Torrero JF, Saponi Cortés JM, Ortiz Descante C, et al. Utilización y eficacia de la vacunación antigripal en la prevención de ingresos hospitalarios por descompensación cardiorrespiratoria en pacientes de alto riesgo en Cáceres. Rev Clin Esp. 2003;203:363-7.

24. Hollmeyer HG, Hayden F, Poland G, Buchholz U. Influenza vaccination of health care workers in hospitals--a review of studies on attitudes and predictors. Vaccine. 2009;27:3935-44.

25. Wicker S, Rabenau HF, Doerr HW, Allwinn R. Influenza vaccination compliance among health care workers in a German university hospital. Infection. 2009;37:197-202.

26. Sánchez-Payá J, Hernández-García I, Barrenengoa-Sañudo J, Camargo-Angeles R, Rincon A, Romero-Candeira S. Frecuencia de reacciones adversas y factores asociados tras la administración de la vacuna de la gripe en personal sanitario durante la temporada 2009-2010. Rev Esp Salud Publica. 2010;84:851-9.

27. Alguacil Ramos AM, Lluch Rodrigo JA, Portero Alonso A, Martín Ivorra R, Pastor Villalba E. Variabilidad en la notificación de reacciones adversas a las vacunas de la gripe pandémica y estacional: Temporadas 2009-2010 y 2010-2011, Comunitat Valenciana. Rev Esp Salud Publica. 2012;86:241-51.

28. Talbot TR. Improving rates of influenza vaccination among healthcare workers: educate; motivate; mandate? Infect Control Hosp Epidemiol. 2008;29:107-10. 
29. Bueno Campaña M, González Spinola A, Parra Cuadrado E, Quevedo Teruel S, Calvo Rey C. Vacunación antigripal en la embarazada. Prog Obstet Ginecol. 2010;53:293-6.

30. Villar Álvarez F. Sesgos y factores de confusión. En: Método epidemiológico. Madrid, Ministerio de Ciencia e Innovación; 2009. p. 47-73.

31. Martínez-González MA, de Irala J. Medicina preventiva y fracaso clamoroso de la salud pública: llegamos mal porque llegamos tarde. Med Clin (Barc). 2005;124:656-60. 